\title{
Management of Idiopathic Pulmonary Fibrosis Cases at a Tertiary Care Hospital
}

\author{
Mahesh NS ${ }^{1}$, Sadanand C D², Durga Lawande ${ }^{3}$ \\ ${ }^{1}$ Assistant Professor, Department of TB and Chest Diseases, Akash Institute of Medical Sciences and Research Centre, Bangalore, Karnataka, ${ }^{2}$ Associate \\ Professor, Department of Medicine, Akash Institute of Medical Sciences and Research Centre, Bangalore, Karnataka, ${ }^{3}$ Professor, Department of \\ Pulmonary Medicine, Goa Medical college, Goa.
}

\section{Abstract}

Background: The clinical history of the disease is quite variable; there is usually a slow physiological deterioration, but in some patients there is a faster decline in lung function and death occurs within 6-12 months after diagnosis. Others experience an acute exacerbation during the course of the disease with a sudden worsening of respiratory symptoms, hypoxemia and the appearance of new radiological infiltrates without an identifiable cause. Despite different types of clinical course, IPF is inevitably associated with a poor prognosis, with a median survival of 2 3 years. Subjects and Methods: Age, sex, occupation, address (including phone number) was noted down. A detailed occupational history was sought, enquiring about the age at start of the occupation, exact nature of work, any history of exposure to fumes, gases, dust, etc, any other person in the work place suffering from similar complaints etc were obtained. Results: Majority of the patients were treated with steroids alone 22(62.8\%). 10(28.5\%) patients were treated with steroids with azathioprine. 3(8.5\%) patients were treated with pirfenidone. Conclusion: Majority of the patients, $17(48.5 \%)$ out of 35 patients, expired during the study period. 5(14.2\%) patients were lost to follow up and the outcome was not available. $10(28.5 \%)$ patients remained stable, while $3(8.5 \%)$ patients worsened. None of the patients improved during the study period.

Keywords: Idiopathic Pulmonary Fibrosis, Pirfenidone, Outcome.

Corresponding Author: Dr. Sadanand C D, Associate Professor, Department of Medicine, Akash Institute of Medical Sciences and Research Centre, Bangalore, Karnataka.

Received: October 2019

Accepted: October 2019

\section{Introduction}

Idiopathic Pulmonary Fibrosis (IPF) is the most common disease in the subgroup of Idiopathic Interstitial Pneumonias. It is a disease of the elderly, with presentation occurring usually in the sixth and seventh decades, more frequently in men. Both etiology and key mechanisms of pathogenesis remain to be understood. IPF's typical histological pattern is Usual Interstitial Pneumonia (UIP). However, in the absence of biopsy, IPF can be diagnosed based on ATS/ERS criteria a group of clinical, radiological and physiological parameters, internationally accepted and validated, in which typical signs at Chest High-Resolution Computerized Tomography (HRCT) have a prominent role. ${ }^{[1]}$

The clinical history of the disease is quite variable; there is usually a slow physiological deterioration, but in some patients there is a faster decline in lung function and death occurs within 6-12 months after diagnosis. ${ }^{[2]}$ Others experience an acute exacerbation during the course of the disease with a sudden worsening of respiratory symptoms, hypoxemia and the appearance of new radiological infiltrates without an identifiable cause. Despite different types of clinical course, IPF is inevitably associated with a poor prognosis, with a median survival of 2-3 years. No proven effective pharmacological therapy has yet been found. ${ }^{[3]}$ Some prognostic factors have already been described, which may have implications for potential therapy, particularly when to refer for lung transplant, which up until now has been the only therapy with demonstrated survival benefit. ${ }^{[4]}$ Recently, Pirfenidone, an oral antifibrotic and antiinflammatory drug, has been approved by EMEA (European Medicines Agency) for the treatment of IPF and is available in India as well.

Our aim was to describe the clinical presentation of IPF patients and to follow up the patients for 1 year duration and to monitor their clinical course and assess them at the end of 1 year for survival. HRCT and PFT'S (Pulmonary Function Test) were done at presentation and after 6 months and after 1 year.

\section{Subjects and Methods}

The diagnosis of IPF requires the following:

1. Exclusion of other known causes of ILD (e.g., domestic and occupational environmental exposures, connective tissue disease, and drug toxicity). 
2. The presence of a UIP pattern on HRCT in patients not subjected to surgical lung biopsy.

3. Specific combinations of HRCT and surgical lung biopsy pattern in patients subjected to surgical lung biopsy.

\section{Inclusion criteria}

- All patients who presented with features suggestive of IPF were subjected to radiological examination with chest radiograph and HRCT thorax.

- Those patients whose HRCT thorax was reported as UIP pattern were included in the study. Surgical lung biopsy was not performed in any of the patients.

- Exclusion criteria

- Patients with history of occupational environmental exposure, drug toxicity were excluded.

- Patients with known connective tissue disease presenting with lung involvement were excluded.

- $\quad$ HRCT thorax reported as findings inconsistent with IPF were excluded.

- Informed consent was obtained from all the patients.

- A pre-structured proforma was filled in those cases which were included in the study.

The following details were included in the study.

1. Personal data: Age, sex, occupation, address (including phone number) was noted down. A detailed occupational history was sought, enquiring about the age at start of the occupation, exact nature of work, any history of exposure to fumes, gases, dust, etc, any other person in the work place suffering from similar complaints etc were obtained.

2. Symptoms: Chief complaints were noted and detailed account of each was obtained. The complaints specifically sought for include cough, dyspnea, other symptoms suggestive of connective tissue disease, like rash, joint pain, raynaud's phenomenon, dysphagia.

3. Past history: Any significant past medical history was noted any co-morbid illness was noted.

4. Family history: Any family history of IPF was asked for.

5. Personal history: Whether the person is or was a smoker. In smokers, age of onset, number of cigarettes/beedis smoke per day and the number of years smoked was obtained. Smoking was quantified in terms of pack years. In case of ex-smokers the number of years after smoking cessation was noted.

6. Physical examination: A complete clinical examination was performed in all cases including general examination, examination of respiratory system in detail and other systems.

7. Investigations: Investigations were done to arrive at the diagnosis and to monitor the patient. Blood investigations to rule out other connective tissue diseases, which were available in the department were done (i.e. ANA, LE Cell, RA factor, ESR, CRP). Routine blood examination- Hb, TC/DC, ESR, FBSL, renal function tests, liver function tests, urine microscopic examination, sputum for AFB etc were done.

Diagnostic Investigations
Chest radiography was performed in all patients.

HRCT was done in all the patients, and was useful to include or exclude the patients from the study, based on the findings reported on HRCT by the radiologist.

Baseline oxygen saturation was recorded in all patients both at rest and after exercise (six minute walk test).

Spirometry was done in most of the patients. Few patients could not do proper PFT'S because of poor respiratory efforts.

$\mathrm{DL}_{\mathrm{CO}}$ and TLC was possible in very few patients included in the study, as the facilities for the same are not available with us in the department.

After diagnosis, the patients were started on treatment. Majority of the patients who utilize our health facility come from poor economic background and cannot afford costly medicines and hence had to be treated with corticosteroid monotherapy. Those affordable patients were started on pirfenidone alone or on azathioprine in combination with steroids.

ATS/ERS joint statement published in 2011, does not propose any consensus on the correct treatment regimen for IPF and hence no particular rule was followed in the choice of medication in each individual patient.

The patients were followed up regularly in monthly intervals, exacerbations were treated with high dose steroids and antibiotics. Chest radiography was done if clinically indicated. HRCT and PFT'S were done at 6 months and after 1 year.

Few patients were lost to follow up during the study and their outcome was not known

\section{Results}

Table 1: Shows chest radiograph findings in the present study

\begin{tabular}{|l|l|l|}
\hline Chest Radiograph & No. Of Patients & Percentage \\
\hline Reticulonodular & 23 & $65.7 \%$ \\
\hline Reticular & 10 & $28.5 \%$ \\
\hline Normal & 3 & $8.5 \%$ \\
\hline
\end{tabular}

Out of the 35 patients, abnormal chest radiograph was found in 32 patients. Majority, 23(65.7\%) of them had reticulonodular abnormality. $10(28.5 \%)$ had reticular abnormality.

Table 2: Shows distribution of lesion in different zone on chest x-ray

\begin{tabular}{|l|l|l|}
\hline Zones & No. Of Patients & Percentage \\
\hline Upper Zone & 1 & $2.8 \%$ \\
\hline Mid Zone & 6 & $17.1 \%$ \\
\hline Lower Zone & 32 & $91.4 \%$ \\
\hline Normal & 3 & $8.5 \%$ \\
\hline
\end{tabular}

32(91.4\%) patients had bilateral lower zone involvement. 6 (17.1\%) patients had lower zone and mid zone lesions bilaterally. 1(2.8\%) patient had upper mid and lower zone involvement.

Table 3: Shows HRCT findings in the present study
\begin{tabular}{|l|l|l|}
\hline Hrct Findings & No. Of Patients & Percentage \\
\hline Interstitial Septal Thickening & 35 & $100 \%$ \\
\hline Honeycombing & 22 & $62.5 \%$ \\
\hline Traction Bronchiectasis & 20 & $57.4 \%$ \\
\hline Parenchymal Distortion & 31 & $88.5 \%$ \\
\hline
\end{tabular}




\begin{tabular}{|l|l|l|}
\hline Ground Glassing & 12 & $34.2 \%$ \\
\hline Parenchymal Nodules & 3 & $8.5 \%$ \\
\hline Emphysema & 4 & $8.5 \%$ \\
\hline Mediastinal Adenopathy & 6 & $17.4 \%$ \\
\hline
\end{tabular}

Out of the 7 patients, 3 patients had TLC between 51-55\% predicted. Highest TLC observed was $79.6 \%$ predicted, and lowest was $48.9 \%$ predicted. Mean TLC was $56.6 \%$ predicted. Single breath helium method was used for estimation of TLC.

Interstitial septal thickening was the commonest abnormality seen on HRCT thorax, being observed in $35(100 \%)$ patients. Other common findings were honeycombing and traction bronchiectasis, seen in 22(62.5\%) patients and 20(57.4\%) patients respectively. Ground glassing was seen in $12(34.2 \%)$ patients. Other abnormalities were parenchymal nodules, emphysematous changes and mediastinaladenopathy.

Table 4: Shows Lobes involved in the present study

\begin{tabular}{|l|l|l|}
\hline Lobes involved & Right lung & Left lung \\
\hline Upper lobe & 7 & 5 \\
\hline Middle lobe & 6 & - \\
\hline Lower lobe & 34 & 34 \\
\hline
\end{tabular}

$34(97.1 \%)$ patients had involvement of both lower lobes. $1(2.8 \%)$ patient had involvement of both upper lobes only. 4(11.4\%) patients had involvement of all the lobes bilaterally.

Table 5: Shows baseline spirometry results in present study

\begin{tabular}{|l|l|}
\hline Type Of Abnormality & Present Study, N=32 \\
\hline Normal & $2(6.2 \%)$ \\
\hline Obstructive & - \\
\hline Restrictive & $30(93.5 \%)$ \\
\hline
\end{tabular}

Baseline spirometry was done in 32 patients. 30(93.5\%) patients had restrictive abnormality. 2(6.2\%) patients had normal curve.

\begin{tabular}{|l|l|}
\hline Table 6: Shows FVC \% PREDICTED results in present study \\
\hline FVC \% Predicted & No. Of Patients \\
\hline$>80 \%$ & $1(3.1 \%)$ \\
\hline $50-79 \%$ & $18(56.2 \%)$ \\
\hline$<50 \%$ & $13(40.6 \%)$ \\
\hline
\end{tabular}

Majority of the patients, 18(56.2\%) patients had FVC \% levels between $50-79 \%$. Mean FVC was $55.4 \%$. 13(40.6\%) patients had FVC\% levels <50\%. Only 1(3.1\%) patient had $\mathrm{FVC} \%>80 \%$.

Table 7: Shows DLco values in present study

\begin{tabular}{|l|l|l|}
\hline DL $\mathbf{c o}$ (\% predicted) & No. Of Patients & Percentage \\
\hline$<15 \%$ & 1 & $2.8 \%$ \\
\hline $16-25 \%$ & 3 & $8.5 \%$ \\
\hline $26-35 \%$ & 2 & $5.7 \%$ \\
\hline$>36 \%$ & 1 & $2.8 \%$ \\
\hline NOT AVAILABLE & 28 & $80 \%$ \\
\hline
\end{tabular}

Diffusion studies were available only in 7 patients. 3(8.5\%) patients had $\mathrm{DL}_{\mathrm{CO}}$ between $16-25 \%$ predicted. Highest $\mathrm{DL}_{\mathrm{CO}}$ was $37.5 \%$ predicted, and the lowest was $14.8 \%$ predicted. Mean DL $\mathrm{DO}_{\mathrm{CO}}$ was $26.1 \%$ predicted.

Table 8: Shows total lung volume values in the present study

\begin{tabular}{|l|l|l|}
\hline TLC (\% Predicted) & No. Of Patients & Percentage \\
\hline $45-50 \%$ & 2 & $5.7 \%$ \\
\hline $51-55 \%$ & 3 & $8.5 \%$ \\
\hline $56-60 \%$ & 1 & $2.8 \%$ \\
\hline$>61 \%$ & 1 & $2.8 \%$ \\
\hline Not Available & 28 & $80 \%$ \\
\hline
\end{tabular}

Table 9: Shows various treatment modalities received by the
patients
\begin{tabular}{|l|l|l|}
\hline Treatment & No. Of Patients & Percentage \\
\hline Pirfenidone & 3 & $8.5 \%$ \\
\hline Steroids & 22 & $62.8 \%$ \\
\hline $\begin{array}{l}\text { Steroids With } \\
\text { Azathioprine }\end{array}$ & 10 & $28.5 \%$ \\
\hline
\end{tabular}

Majority of the patients were treated with steroids alone $22(62.8 \%)$. 10(28.5\%) patients were treated with steroids with azathioprine. 3(8.5\%) patients were treated with pirfenidone.

Table 10: Shows follow up results at the end of study period

\begin{tabular}{|l|l|l|}
\hline Follow Up & No. Of Patients & Percentage \\
\hline Stable & 10 & $28.5 \%$ \\
\hline Improved & 0 & 0 \\
\hline Worsened & 3 & $8.5 \%$ \\
\hline Expired & 17 & $48.5 \%$ \\
\hline Lost For Follow Up & 5 & $14.2 \%$ \\
\hline Total & 35 & $100 \%$ \\
\hline
\end{tabular}

Majority of the patients, 17(48.5\%) out of 35 patients, expired during the study period. 5(14.2\%) patients were lost to follow up and the outcome was not available. 10(28.5\%) patients remained stable, while $3(8.5 \%)$ patients worsened. None of the patients improved during the study period.

Table 11: Shows individual variables in the expired and not expired group.

\begin{tabular}{|l|l|l|}
\hline Variable & $\begin{array}{l}\text { Expired patients, } \\
\mathbf{n = 1 7}\end{array}$ & $\begin{array}{l}\text { Not expired, } \\
\mathbf{n = 1 3}\end{array}$ \\
\hline Age in years (Mean) & 62.5 & 61.9 \\
\hline Sex M = Male, F = Female & $\mathrm{M}=8, \mathrm{~F}=9$ & $\mathrm{M}=6, \mathrm{~F}=7$ \\
\hline Smoker (current or ex) & 12 & 5 \\
\hline Clubbing (present) & 10 & 7 \\
\hline FVC\% predicted, $\mathrm{n}=32$ (Mean) & $52.9 \%$ & $58.5 \%$ \\
\hline Baseline resting saturation & $91.1 \%$ & $95.6 \%$ \\
\hline $\begin{array}{l}\text { Exercise desaturation (6 MWT) } \\
\text { (Present), } \mathrm{n}=32\end{array}$ & 12 & 2 \\
\hline
\end{tabular}

At the end of 1 year follow up 17 patients are expired and 5 patients were lost to follow up. The above mentioned parameters were compared in the expired and not expired group. Patients were lost to follow up were not included in the comparison.

32 patients were able to perform 6MWT and Spirometrymanoevres. 3 patients could not do these tests because of severe respiratory a compromise.

\section{Discussion}

Virtually all patients with IPF have an abnormal chest radiograph at the time of presentation. The most common radiographic finding in IPF, described in approximately $80 \%$ of patients consists of bilateral irregular linear opacities causing a reticular pattern. Although these opacities may be diffuse they involve predominantly the lower lung zones. As 
fibrosis progresses, the reticular pattern becomes coarser, and there is progressive loss of lung volume. Honeycombing represents end-stage fibrosis. The plain radiographic appearance of IPF is nonspecific and misleading, being similar to that seen in many other interstitial lung diseases. Furthermore, it has been repeatedly demonstrated that the severity and extent of the disease assessed on the chest radiograph correlates poorly with the functional and clinical impairment. The clinical utility and optimal timing of followup chest radiographs are unclear. Radiographs are indicated if clinical deterioration occurs, to assess progression of disease, or to identify superimposed infection or malignancy. In the present study on the chest $\mathrm{x}$-ray the commonest finding was reticulonodular lesions seen in 23(65.7\%) patients, predominantly involving the lower zones and mid zones.

The results were comparable with the study done by maheshwariet $\mathrm{al}^{[5]}$, who reported predominant reticulonodular shadows on chest radiograph in 45(59.2\%) patients out of 70 .

Three patients who had normal chest radiograph, had history and clinical examination suggestive of IPF, were subjected to further evaluation by HRCT thorax and were diagnosed to have IPF based on characteristic CT picture.

The predominant HRCT features of IPF include honeycombing and intralobular interstitial thickening; irregular interlobular septal thickening and ground glass opacity may also be present but are usually less conspicuous findings. The abnormalities are preferentially in the peripheral, subpleural region and lower lung zone and posterior predominance is usual. ${ }^{[6]}$

Another hallmark of IPF is patchy distribution. Areas of mild and severe fibrosis, inflammatory activity, and normal lung are often present in the same patient, in the same lung, and in the same lobe subpleural region and lower lung zone and posterior predominance is usual. ${ }^{[6]}$

The accuracy of a confident diagnosis of UIP made on HRCT by a trained observer appears to be about $90 \%$. ${ }^{[6]}$

In our study, all the patients were diagnosed as IPF, based on the characteristic UIP pattern on HRCT scan. Lung biopsy was not done in any of the patients.

The distribution of features seen on HRCT observed in our study is compared below with similar studies done in the past. In the present study, there was peripheral and lower lobe predominance of the lesions.

Distribution of lesions was often bilateral in most patients. 4(8.5\%) patients showed involvement of all lobes and 1 (2.8\%) had involvement of only upper lobes in both lungs.

The interstitial septal thickening is the most common finding on HRCT in patients with IPF in $96 \%$ of cases results in fine reticular pattern. Thickening of interstitial interstitium also results in the presence of irregular interfaces between the lung and pulmonary vessels, bronchi, and pleural surfaces. In areas of severe fibrosis, the segmental \&subsegmental bronchi show traction bronchiectasis. ${ }^{[7]}$

In our study, interstitial septal thickening and traction bronchiectasis were seen in $100 \%$ and $57.4 \%$ of cases respectively.

Honeycombing is another common finding associated with gross distortion of lung architecture, where individual lobules are no longer visible. Honeycomb cyst usually range from 2 to $20 \mathrm{~mm}$ in diameter, appear to share walls on HRCT, occurs in subpleural lung and in several layers.Finding of honeycombing and fibrosis are most often bilaterally symmetrical. ${ }^{[8]}$

Honeycombing occurs in $24-90 \%$ of patients who have IPF, and the frequency of this finding varies with the severity or stage of the disease. In our study it was seen in $(62.5 \%)$ of cases, but Maheshwariet $\mathrm{al}^{[5]}$ reported this finding in $90.8 \%$ cases.

IPF presenting with GGO (Ground glass opacity) as a predominant finding is distinctly uncommon. Presence of GGO as a predominant findings need further evaluation with lung biopsy. ${ }^{[6]}$

In our study GGO was seen in only $12(34.2 \%)$ cases and in these patients it was not a predominant finding. This possibly represents our study population in advanced stage of disease. Mediastinal lymphadenopathy usually less than $15 \mathrm{~mm}$ in short axis diameter \& involving one or two nodal stations is seen in 46- $90 \%$ of patients of IPF. The likelihood of lymphadenopathy increases with the extent of parenchymal involvement and decreases in the presence of recent steroid treatment. ${ }^{[6]}$

In our study 6 (17.4\%) patients had mediastinaladenopathy. As such, pulmonary function tests (PFTs) should be used in conjunction with clinical, radiographic, and histologic information in assuring an accurate diagnosis. On the other hand, in patients with appropriate symptoms, PFTs can serve as early diagnostic tools.

Treatment options include corticosteroids, immunosuppressive/cytotoxic agents (e.g. azathioprine, cyclophosphamide), and antifibrotic agents (e.g. colchicine or D-penicillamine, pirfenidone) alone or in combination.

No randomized controlled trials have been conducted with corticosteroid monotherapy. Retrospective uncontrolled studies have reported no survival benefits, but have suggested that a minority of patients treated with corticosteroid monotherapy improve their pulmonary function; controlled data have found no survival benefit. There is substantial morbidity from long-term corticosteroid therapy. ${ }^{[3]}$

A retrospective study suggested a potential benefit of treatment with azathioprine plus prednisone in a small case series. ${ }^{[9]}$

A small randomized trial of corticosteroid versus corticosteroid and azathioprine showed a trend toward a survival benefit with combination therapy with corticosteroid and azathioprine.

Pirfenidone is a pyridone compound with pleiotropic, antiinflammatory, antifibrotic, and antioxidant properties, with antagonism of TGF-b1 effects. A randomized controlled trial (RCT) from Japan comparing pirfenidone with placebo was stopped prematurely after a secondary endpoint, acute exacerbation was found significantly more frequently in the placebo group as compared with the active treatment arm. This study demonstrated beneficial treatment effect on oxygen saturation during 6-minute steady-state exercise test (which was the primary endpoint) and a significantly diminished decline of vital capacity in the active treatment compared with the placebo arm.

A second RCT from Japan comparing pirfenidone to placebo found a reduction in the rate of decline in vital capacity over 52 weeks in the pirfenidone arm. There was also a difference in progression-free survival (defined by death or $10 \%$ decline 
in vital capacity) favoring the pirfenidone group.

The bottom line is that, at present there is not a single drug that is effective in the treatment of IPF. Extensive research is going on in this area all over the world. In the future, hopefully we may find the molecule which is effective in the treatment of this condition.

Majority of the patients who use our health facility are from economically poor background and cannot afford costly medication and hence were being treated with steroid alone as monotherapy.

In our study, 22(62.8\%) patients received steroids as monotherapy. Oral prednisolone was started at the dose of $0.5 \mathrm{mg} / \mathrm{kg}$ body weight daily for 4 weeks, $0.25 \mathrm{mg} / \mathrm{kg}$ daily for 8 weeks, followed by $0.25 \mathrm{mg} / \mathrm{kg}$ every other day.

$10(28.5 \%)$ were treated with azathioprine and steroid. Azathoprine was started at a dose of $50 \mathrm{mg}$ per day orally, increasing by $25 \mathrm{mg}$ increments every 14 days and maintained at $100 \mathrm{mg}$ per day.

3 patients in the study were started on pirfenidone. They were initially started at a dose of $600 \mathrm{mg}$ per day, the dose was gradually increased to $1200 \mathrm{mg}$ per day after 4 weeks.

There appear to be several possible natural histories for patients with IPF. Natural history is unpredictable at the time of the diagnosis. The majority of patients demonstrate a slow, gradual progression over many years. Some patients remain stable while others have an accelerated decline. Some patients may experience episodes of acute respiratory worsening. Other comorbid conditions such as emphysema and pulmonary hypertension may impact the disease course. ${ }^{3}$ In our study all the patients were followed up for the period of 1 year. Follow up HRCT thorax and spirometry was repeated after 6 months of presentation and after 1 year in our study.

9 patients had expired within 6 months of presentation and 3 patients were lost to follow up. PFT'S and HRCT were done in remaining patients.

3 patients complained of progressive dyspnea (grading by MMRC classification), none of them had any significant change radiologically. One patient had $>10 \%$ reduction in $\mathrm{FVC} \%$ predicted, when compared to previous value.

Acute exacerbation of IPF is defined as unexplained worsening of dyspnea within 1 month, evidence of hypoxemia as defined by worsened or severely impaired gas exchange, new radiographic alveolar infiltrates, and an absence of an alternative explanation such as infection, pulmonary embolism, pneumothorax, or heart failure. ${ }^{3}$

In our study, $12(40 \%)$ patients had no exacerbations in 1 year follow up period.

Other patients had $1-3$ exacerbations in the one year duration. One patient had 4 exacerbations.

Patients were treated with high dose steroids, antibiotics and supportive care.

In studies that used the revised diagnostic criteria for IPF, only 20 to $30 \%$ of subjects were alive 5 years after diagnosis. There are many individual variables that have been shown to predict survival in IPF. In general, factors that are associated with shortened survival time include: older age, smoking history, more severe physiologic impairment, greater radiologic extent of disease, and the development of other complications or conditions, in particular, pulmonary hypertension, emphysema, and bronchogenic cancer. ${ }^{10}$

In the present study it was observed that 17 (48.5\%) patients expired with in 1 year after diagnosis.

The major limitation in this analysis is that DLco (measures physiological imapirment), radiological extent of the disease and co morbidities were not included in the analysis. Other variables may become significant if these factors were introduced in the analysis.

The follow up period in the study was only 1 year, which is relatively less. Further follow up of patients for up to 3 to 5 years is required for proper analysis of mortality rate.

\section{Conclusion}

To conclude, similar to the diagnosis of IPF, prognosis of individual patients will likely best be determined in the future using a multidisciplinary approach. Such an approach should incorporate information from multiple areas of clinical care using a well-developed and validated clinical prediction model.

This will allow clinicians to better manage patients and provide a valuable tool for future clinical trials.

\section{References}

1. Kim DS, Collard HR, King Jr TE. Classification and natural history of idiopathic interstitial pneumonias. Proc Am Thorac Soc. 2006; 3:285-92.

2. American Thoracic Society, European Respiratory Society. American Thoracic Society/European Respiratory Society International Multidisciplinary Consensus Classification of the Idiopathic Interstitial Pneumonias. Am J RespirCrit Care Med. 2002;165:277304.

3. Raghu G, Collard HR, Egan JJ, Martinez FJ, Behr J, Brown KK, et al. An official ATS/ERS/JRS/ALAT statement: idiopathic pulmonary fibrosis: evidence-based guidelines for diagnosis and management. Am J RespirCrit Care Med. 2011;183: 788-824.

4. Fernández Pérez ER, Daniels CE, Schroeder DR, St Sauver J, Hartman TE, Bartholmai BJ, et al. Incidence, prevalence and clinical course of idiopathic pulmonary fibrosis. Chest. 2010;137:129-37.

5. Maheshwari U, Gupta D, Aggarwal A.N, Jindal S.K. Spectrum and diagnosis of Idiopathic pulmonary fibrosis. Indian T.Chestdis Allied Sci. 2004; 46: 23-26.

6. S Raniga, S Sharma, AArora, Y Khalasi, Pa Vora. Utility of High Resolution Computed Tomography (HRCT) in diagnosis and management of Idiopathic Pulmonary Fibrosis - A study of 10 cases". Ind J RadiolImag. 2006;16:4:841-46.

7. Wells, A. U., P. Cullinan, D. M. Hansell, M. B. Rubens, C. M. Black, A. J. Newman-Taylor, and R. M. Du Bois. Fibrosingalveolitis associated with systemic sclerosis has a better prognosis than lone cryptogenic fibrosingalveolitis..Am J. Respir. Crit. Care Med.1994;149:1583-90.

8. Cherniack, R. M., T. V. Colby, A. Flint, W. M. Thurlbeck, J. A. Waldron, Jr., L. Ackerson, M. I. Schwarz, and T. E. King, Jr. Correlation of structure and function in idiopathic pulmonary fibrosis. Am.J. Respir. Crit. Care Med.1995;151:1180-88.

9. F. SoaresPires, P. Caetano Mota, N. Melo, D. Costa, J.M. Jesus, R. Cunha, S. Guimarães, C. Souto-Moura, A. Morais . Idiopathic Pulmonary Fibrosis-Clinical presentation, outcome and baseline prognostic factors in a Portuguese cohort. Rev Port Pneumol. 2013;19(1):19-27.

10. Katzenstein, A.-L. A., J. L. Myers, W. D. Prophet, L. S. Corley, III, and M. S. Shin. Bronchiolitis obliterans and usual interstitial pneumonia: a comparative clinicopathologic study. Am. J. Surg. Pathol. 1986;106: 373-81. 
Copyright: () the author(s), 2019. It is an open-access article distributed under the terms of the Creative Commons Attribution License (CC BY 4.0), which permits authors to retain ownership of the copyright for their content, and allow anyone to download, reuse, reprint, modify, distribute and/or copy the content as long as the original authors and source are cited.

How to cite this article: Mahesh NS, Sadanand C D, Lawande D. Management of Idiopathic Pulmonary Fibrosis Cases at a Tertiary Care Hospital. Acad. J Med. 2019;2(2):90-95.

DOI: dx.doi.org/10.21276/ajm.2019.2.2.23 